TRI-PP-05-25, USC(NT)-05-06

\title{
Neutron-Neutron Fusion
}

\author{
Shung-ichi Ando ${ }^{a, 1}$ and Kuniharu Kubodera ${ }^{b, 2}$ \\ ${ }^{a}$ Theory Group, TRIUMF, 4004 Wesbrook Mall, Vancouver, B.C. V6T 2A3, Canada \\ ${ }^{b}$ Department of Physics and Astronomy, University of South Carolina, Columbia, SC \\ 29208, USA
}

The neutron-neutron fusion process, $n n \rightarrow d e \nu$, at very low neutron energies is studied in the framework of pionless effective field theory that incorporates dibaryon fields. The cross section and electron energy spectrum for this process are calculated up to nextto-leading order. We include the radiative corrections of $\mathcal{O}(\alpha)$ calculated for the onebody transition amplitude. The precision of our theoretical estimates is found to be governed essentially by the accuracy with which the empirical values of the neutronneutron scattering length and effective range are currently known. Also discussed is the precision of theoretical estimates of the transition rates of related electroweak processes in few-nucleon systems.

\footnotetext{
${ }^{1}$ E-mail:sando@triumf.ca

${ }^{2}$ E-mail:kubodera@sc.edu
} 


\section{Introduction}

The ultra-high-intensity neutron-beam facilities currently under construction at, e.g., the Oak Ridge National Laboratory and the J-PARC are expected to bring great progress in high-precision experiments concerning the fundamental properties of the neutron and neutron $\beta$-decay. The planned experiments of great importance include the accurate determination of the neutron electric dipole moment, and high-precision measurements of the lifetime and the correlation coefficients for neutron $\beta$-decay. Besides these experiments that focus on the properties of a single neutron, one might be tempted to consider processes that involve the interaction of two free neutrons. An example is the direct observation of free neutron-neutron scattering, which would allow the model-independent determination of the neutron-neutron scattering length $a_{0}^{n n}$ and effective range $r_{0}^{n n}$. These quantities play an important role in high-precision calculations of low-energy electroweak transitions involving the $n n$ channel (see below). Their accurate values are also important in the study of isospin symmetry breaking effects in the strong interactions $[1,2]$. Up to now, however, because of the lack of free neutron targets, information on $a_{0}^{n n}$ and $r_{0}^{n n}$ has been obtained by analyzing processes such as $\pi^{-} d \rightarrow n n \gamma$ or $n d \rightarrow n n p$; for the current status of these experiments, see, e.g., Refs. [3, 4, 5]. Although there has been considerable improvement in these analysis, they still contain rather significant theoretical uncertainties. For instance, the treatments of the exchange currents and higher partial waves in the $\pi^{-} d \rightarrow n n \gamma$ process [6] are open to further examinations; similarly, the treatment of the three-body interactions in the $n d \rightarrow n n p$ reaction is yet to be settled. Therefore, the observation of free neutron-neutron scattering is an interesting alternative, even though its extremely low counting rate is certainly a major obstacle. ${ }^{3}$

As another process involving two free neutrons, we consider here the neutron-neutron fusion reaction

$$
n+n \rightarrow d+e^{-}+\bar{\nu}_{e}
$$

for neutrons of very low energies such as the ultra-cold neutrons and thermal neutrons. The experimental observation of this reaction does not seem to belong to the near future but, in view of the existing strong thrust for pursuing experiments that take advantage of ultra-high-intensity neutron beams, it may not be totally purposeless to make a theoretical study of $n n$-fusion, and this is what we wish to do here. It is worth noting that, for very low energy neutrons, the maximum energy $E_{e}^{\max }$ of the outgoing electron is $E_{e}^{\max } \simeq B+\delta_{N} \simeq$ $3.52 \mathrm{MeV}$, where $B$ is the deuteron binding energy and $\delta_{N}$ is the mass difference between the neutron and proton, $\delta_{N}=m_{n}-m_{p}$. This value of $E_{e}^{\max }$ is significantly larger than the maximum energy of electrons from neutron $\beta$-decay, $E_{e, \beta \text {-decay }}^{\max } \simeq \delta_{N} \simeq 1.29 \mathrm{MeV}$. Thus the $n n$-fusion electrons with energies larger than $\delta_{N}$ are in principle distinguishable from the main background of the neutron $\beta$-decay electrons.

Our study is based on low-energy effective field theory (EFT). For a system of mesons and baryons (without or with external probes), EFT provides a systematic perturbative

\footnotetext{
${ }^{3}$ There exists a project to detect the scattering of two free neutrons using reactor-generated neutrons [7].
} 
expansion scheme in terms of $Q / \Lambda$, where $Q$ denotes a typical momentum scale of the process under consideration, and $\Lambda$ represents a cutoff scale that characterizes the EFT lagrangian [8]. In a higher order effective lagrangian, there appear unknown parameters, so-called low energy constants (LECs), which represent the effects of high-energy physics that has been integrated out. In many cases, these LECs cannot be determined by the symmetries of the theory alone and hence need to be fixed by experiments. Once all the relevant LECs are fixed, the EFT lagrangian represents a complete (and hence modelindependent) lagrangian to a given order of expansion.

Many studies based on EFT have been made of nuclear processes in few-nucleon systems; for reviews see, e.g., Refs. [9, 10,11] and references therein. These studies have used various versions of EFT that differ primarily in the treatment of the following points. (1) Choice between the Weinberg counting scheme [12] and the KSW counting scheme [13]; (2) Inclusion or exclusion of the pion field as an active degree of freedom [14]; (3) The presence of an abnormally low energy scale reflecting the large scattering lengths in the nucleonnucleon interaction is handled by introducing an expansion of the inverse of the scattering amplitude [13], or by introducing the effective dibaryon fields (see, e.g., Ref. [15]). (For convenience, we refer to the former as the $1 / \mathcal{A}$ expansion method, and the latter as dEFT.) For the reasons to be explained below, we use here pionless dEFT.

The $n n$-fusion process of our concern here is closely related to the $p p$-fusion reaction $\left[p p \rightarrow d e^{+} \nu\right]$, and the $\nu d$ reactions $\left[\nu d \rightarrow p p e(n p \nu), \bar{\nu} d \rightarrow n n e^{+}(n p \bar{\nu})\right]$, which have been studied extensively because of their importance in astrophysics and neutrino physics; see, e.g., Refs. [16, 17, 18, 19] for $p p$-fusion, and Refs. [20, 21, 22, 23, 24] for the $\nu d$ reactions. In an EFT treatment of these reactions, there occurs a LEC that controls the strength of contact two-nucleon-axial current coupling. This LEC is referred to as $\hat{d}^{R}$ in Weinbergscheme calculations [19] and as $L_{1 A}$ in KSW-scheme calculations [23]. Park et al. have developed a useful hybrid approach called EFT* to determine $\hat{d}^{R}[19]{ }^{4}$ In EFT*, the nuclear transition amplitude for an electroweak process is calculated with the use of the relevant transition operator derived from EFT along with the nuclear wave functions obtained from a high-precision phenomenological nucleon-nucleon potential. ${ }^{5}$ An advantage of EFT $^{*}$ is that it can be used for light complex nuclei $(\mathrm{A}=3,4, \ldots)$ with essentially the same accuracy and ease as for the two-nucleon systems. The use of EFT* enabled Park et al. to determine $\hat{d}^{R}$ with good accuracy from the tritium $\beta$-decay rate [19]. ${ }^{6}$ This result allowed parameter-free calculations of the $p p$-fusion and $H e p$-reaction rates [19], and the $\nu d$-reaction cross sections [24]. It is certainly possible to extend this EFT* calculation to the $n n$-fusion process, but we do not attempt it here. Instead, we employ pionless dEFT with motivations to be described below.

As is well known, the EFT treatment of low-energy nucleon-nucleon scattering is complicated by the existence of a large length scale associated with the very-weakly bound state (deuteron) in the ${ }^{3} S_{1^{-}}{ }^{3} D_{1}$ channel, or with the near-threshold resonance (virtual

\footnotetext{
${ }^{4}$ This approach is also called MEEFT (more effective EFT); for a review, see, e.g., Ref. [11].

${ }^{5}$ For discussion of some formal aspects of EFT*, see Ref. [25].

${ }^{6}$ Attempts to determine $L_{1 A}$ can be found in, e.g., Refs. [26, 27, 28]; for the possible use of $\mu d$ capture to determine $\hat{d}^{R}$ or $L_{1 A}$, see Refs. [29, 30,31].
} 
state, or singlet deuteron) in the ${ }^{1} S_{0}$ channel. The $1 / \mathcal{A}$-expansion method was proposed to cope with this difficulty. This method, however, exhibits rather slow convergence in the deuteron channel, for which a typical expansion parameter is $\left(\gamma \rho_{d}\right) \simeq 0.4 \sim 1 / 3$, where $\gamma \simeq 45.7 \mathrm{MeV}$ and $\rho_{d} \simeq 1.764 \mathrm{fm}$. Thus, to achieve $1 \%$ or $3 \%$ accuracy, one must go up to next-to-next-to-next-to-next-to-leading order $\left(\mathrm{N}^{4} \mathrm{LO}\right)$ or next-to-next-tonext-to-leading $\left(\mathrm{N}^{3} \mathrm{LO}\right)$ order $\left((1 / 3)^{4} \sim 1 \%\right.$ or $\left.(1 / 3)^{3} \sim 3 \%\right)$. These $\mathrm{N}^{4} \mathrm{LO}$ and $\mathrm{N}^{3} \mathrm{LO}$ calculations are formidably challenging even in the pionless cases. ${ }^{7}$ Another method to resolve the difficulty associated with the existence of the large length scale in nucleonnucleon scattering is to introduce explicit $S$-channel states, called the "dibaryons", which represent the near-threshold resonance state for the ${ }^{1} S_{0}$ channel, and the deuteron state in the ${ }^{3} S_{1^{-}}{ }^{3} D_{1}$ channel $[35,36]$. (As mentioned earlier, we refer to an EFT that includes the dibaryon fields as dEFT.) Beane and Savage [15] discussed counting rules in dEFT and moreover demonstrated the usefulness of dEFT in describing the electromagnetic processes in the two-nucleon systems. Furthermore, a recent study [37] of the electroweak observables of the deuteron shows that dEFT allows one to achieve in a very transparent and economical way the level of accuracy that, in the $1 / \mathcal{A}$-expansion method, would require the inclusion of terms of very high orders.

The above consideration motivates us to use dEFT in studying the $n n$-fusion process. We limit ourselves here to the case of very low incident neutron energy, which makes it safe to eliminate the pion fields and concentrate on pionless dEFT. We calculate the total cross section and electron energy spectrum for $n n$-fusion up to NLO in dEFT. Although the enormous difficulty of observing $n n$-fusion may render it unwarranted to pursue high precision in our calculation, we still wish to aim at a few percent accuracy for the following reason. As mentioned, $n n$-fusion is closely related to $p p$-fusion and the $\nu d$ reaction, and the latter two processes do require high-precision calculations. Since our present formalism can be applied (with essentially no change) to high-precision estimation of the $p p$-fusion and $\nu d$ cross sections, we may use the $n n$-fusion case to explain what is involved in those high-precision calculations. It turns out (see below) that, at the level of a few percent accuracy, we need to treat properly: (1) the LEC, $l_{1 A}$, which represents the strength of a dibaryon-dibaryon-axial-vector $(d d A)$ interaction and which is associated with $\hat{d}^{R}$ and $L_{1 A}$ discussed earlier; (2) the radiative corrections; (3) the influence of uncertainties in the currently available experimental information on $a_{0}^{n n}$ and $r_{0}^{n n}$. We will show that main uncertainties in our calculation of the low-energy $n n$-fusion cross section come from the last item (3).

\section{Effective lagrangian}

For low-energy processes, the weak-interaction Hamiltonian can be taken to be

$$
\mathcal{H}=\frac{G_{F} V_{u d}}{\sqrt{2}} l_{\mu} J^{\mu},
$$

where $G_{F}$ is the Fermi constant and $V_{u d}$ is the CKM matrix element. $l_{\mu}$ is the lepton

\footnotetext{
${ }^{7}$ It has been suggested by Rho and other authors that much better convergence is achieved by adjusting the deuteron wave function to fit the asymptotic $S$-state normalization constant, $Z_{d}=\gamma \rho_{d} /\left(1-\gamma \rho_{d}\right)[32$, $33,34]$.
} 
current $l_{\mu}=\bar{u}_{e} \gamma_{\mu}\left(1-\gamma_{5}\right) v_{\nu}$, while $J_{\mu}$ is the hadronic current, which we calculate here up to two-body terms based on the effective lagrangian of dEFT.

We adopt the standard counting rules of dEFT [15]. Introducing an expansion scale $Q<\Lambda\left(\simeq m_{\pi}\right)$, we count the magnitude of spatial part of the external and loop momenta, $|\vec{p}|$ and $|\vec{l}|$, as $Q$, and their time components, $p^{0}$ and $l^{0}$, as $Q^{2}$. The nucleon and dibaryon propagators are of $Q^{-2}$, and a loop integral carries $Q^{5}$. The scattering lengths and effective ranges are counted as $Q \sim\left\{\gamma, 1 / a_{0}, 1 / \rho_{d}, 1 / r_{0}\right\}$. The orders of vertices and transition amplitudes are easily obtained by counting the numbers of these factors in the lagrangian and diagrams, respectively. As discussed below, some vertices acquire factors like $r_{0}$ and $\rho_{d}$ after renormalization and thus their orders can differ from what the above naive dimensional analysis suggests.

A pionless dEFT lagrangian may be written as $[15,37]$

$$
\mathcal{L}=\mathcal{L}_{N}+\mathcal{L}_{s}+\mathcal{L}_{t}+\mathcal{L}_{s t},
$$

where $\mathcal{L}_{N}$ is a one-nucleon lagrangian, $\mathcal{L}_{s}$ is the spin-singlet dibaryon lagrangian including coupling to the nucleon, $\mathcal{L}_{t}$ is the spin-triplet dibaryon lagrangian including coupling to the nucleon; $\mathcal{L}_{s t}$ describes the weak-interaction transition (due to the axial current) from the ${ }^{1} S_{0}$ dibaryon to the ${ }^{3} S_{1}$ dibaryon. A pionless one-nucleon lagrangian in the heavybaryon formalism reads

$$
\mathcal{L}_{N}=N^{\dagger}\left\{i v \cdot D-2 i g_{A} S \cdot \Delta+\frac{1}{2 m_{N}}\left[(v \cdot D)^{2}-D^{2}\right]+\cdots\right\} N,
$$

where the ellipsis represents terms that do not appear in this calculation. $v^{\mu}$ is the velocity vector satisfying $v^{2}=1$; we choose $v^{\mu}=(1, \overrightarrow{0}) . S^{\mu}$ is the spin operator $2 S^{\mu}=(0, \vec{\sigma})$, while $D_{\mu}=\partial_{\mu}-\frac{i}{2} \vec{\tau} \cdot \overrightarrow{\mathcal{V}}_{\mu}$ where $\overrightarrow{\mathcal{V}}_{\mu}$ is the external isovector vector current; $\Delta_{\mu}=-\frac{i}{2} \vec{\tau} \cdot \overrightarrow{\mathcal{A}}_{\mu}$, where $\overrightarrow{\mathcal{A}}_{\mu}$ is the external isovector axial current. $g_{A}$ is the axial-vector coupling constant, and $m_{N}$ is the nucleon mass. The terms that involve the dibaryon fields are given by

$$
\begin{aligned}
\mathcal{L}_{s} & =\sigma_{s} s_{a}^{\dagger}\left[i v \cdot D+\frac{1}{4 m_{N}}\left[(v \cdot D)^{2}-D^{2}\right]+\Delta_{s}\right] s_{a}-y_{s}\left[s_{a}^{\dagger}\left(N^{T} P_{a}^{\left({ }^{1} S_{0}\right)} N\right)+\text { h.c. }\right], \\
\mathcal{L}_{t} & =\sigma_{t} t_{i}^{\dagger}\left[i v \cdot D+\frac{1}{4 m_{N}}\left[(v \cdot D)^{2}-D^{2}\right]+\Delta_{t}\right] t_{i}-y_{t}\left[t_{i}^{\dagger}\left(N^{T} P_{i}^{\left({ }^{3} S_{1}\right)} N\right)+\text { h.c. }\right], \\
\mathcal{L}_{s t} & =-\left[\left(\frac{r_{0}+\rho_{d}}{2 \sqrt{r_{0} \rho_{d}}}\right) g_{A}+\frac{l_{1 A}}{m_{N} \sqrt{r_{0} \rho_{d}}}\right]\left[s_{a}^{\dagger} t_{i} \mathcal{A}_{i}^{a}+\text { h.c. }\right],
\end{aligned}
$$

where $s_{a}$ and $t_{i}$ are the dibaryon fields for the ${ }^{1} S_{0}$ and ${ }^{3} S_{1}$ channel, respectively. The covariant derivative for the dibaryon field is given by $D_{\mu}=\partial_{\mu}-i C \mathcal{V}_{\mu}^{e x t}$ where $\mathcal{V}_{\mu}^{\text {ext }}$ is the external vector field. $C$ is the charge operator for the dibaryon field; $C=0,1,2$ for the $n n, n p, p p$ channel, respectively. $\sigma_{s, t}$ is the sign factor and $\Delta_{s, t}$ is the mass difference between the dibaryon and two nucleons, $m_{s, t}=2 m_{N}+\Delta_{s, t} . r_{0}$ and $\rho_{d}$ are the effective ranges for the deuteron and ${ }^{1} S_{0}$ state, respectively. $P_{i}^{(S)}$ is the projection operator for the $S={ }^{1} S_{0}$ or ${ }^{3} S_{1}$ channel;

$$
P_{a}^{\left({ }^{1} S_{0}\right)}=\frac{1}{\sqrt{8}} \sigma_{2} \tau_{2} \tau_{a}, \quad P_{i}^{\left({ }^{3} S_{1}\right)}=\frac{1}{\sqrt{8}} \sigma_{2} \sigma_{i} \tau_{2}, \quad \operatorname{Tr}\left(P_{i}^{(S) \dagger} P_{j}^{(S)}\right)=\frac{1}{2} \delta_{i j},
$$




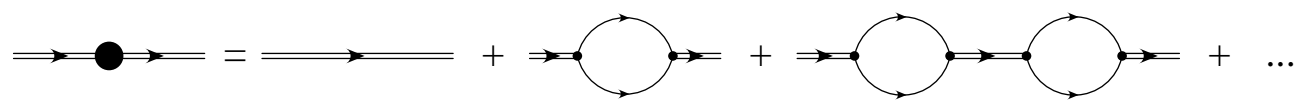

Figure 1: Dressed dibaryon propagator (double line with a filled circle) at leading order. A single line stands for the nucleon, while a double line represents the bare dibaryon.

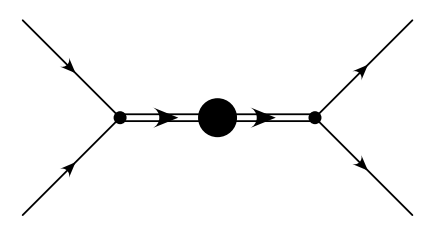

Figure 2: Diagram for the $S$-wave $N N$ scattering amplitude at leading order. The double line with a filled circle represents the dressed dibaryon propagator obtained in Fig. 1.

where $\sigma_{i}\left(\tau_{a}\right)$ is the spin (isospin) operator.

The LECs, $y_{s}$ and $y_{t}$, represent the dibaryon- $N N(d N N)$ couplings in the spin-singlet and spin-triplet states, respectively. These LECs along with $\Delta_{s, t}$ and $\sigma_{s, t}$ are to be determined from the effective ranges in the ${ }^{1} S_{0}$ and ${ }^{3} S_{1}$ channels. LO diagrams for the dressed dibaryon propagators are depicted in Fig. 1. Since an insertion of the two-nucleon one-loop diagram does not alter the order of the diagram, the two-nucleon bubbles should be summed up to infinite order. Thus the inverse of the dressed dibaryon propagator in the center-of-mass $(\mathrm{CM})$ frame reads

$$
\begin{aligned}
i D_{s, t}^{-1}(p) & =i \sigma_{s, t}\left(E+\Delta_{s, t}\right)+i y_{s, t}^{2} \frac{m_{N}}{4 \pi}(i p) \\
& =i \frac{m_{N} y_{s, t}^{2}}{4 \pi}\left[\frac{4 \pi \sigma_{s, t} \Delta_{s, t}}{m_{N} y_{s, t}^{2}}+\frac{4 \pi \sigma_{s, t} E}{m_{N} y_{s, t}^{2}}+i p\right]
\end{aligned}
$$

where we have used dimensional regularization for the loop integral and $E$ is the total energy of the two nucleons, $E \simeq p^{2} / m_{N}$. The dressed dibaryon propagators are renormalized via the $S$-wave $N N$ scattering amplitudes. The amplitudes obtained from the diagram in Fig. 2 should satisfy

$$
i A_{s, t}=\left(-i y_{s, t}\right)\left(i D_{s, t}(p)\right)\left(-i y_{s, t}\right)=\frac{4 \pi}{m_{N}} \frac{i}{-\frac{4 \pi \sigma_{s, t} \Delta_{s, t}}{m_{N} y_{s, t}^{2}}-\frac{4 \pi \sigma_{s, t}}{m_{N} y_{s, t}^{2}} p^{2}-i p},
$$

where $A_{s, t}$ is related to the $S$-wave NN scattering $S$-matrix via

$$
S-1=e^{2 i \delta_{s, t}}-1=\frac{2 i p}{p \cot \delta_{s, t}-i p}=i\left(\frac{p m_{N}}{2 \pi}\right) A_{s, t},
$$


Here $\delta_{s}\left(\delta_{t}\right)$ is the phase shift for the ${ }^{1} S_{0}\left({ }^{3} S_{1}\right)$ channel. Meanwhile, effective range expansion reads

$$
p \cot \delta_{s}=-\frac{1}{a_{0}}+\frac{1}{2} r_{0} p^{2}+\cdots, \quad p \cot \delta_{t}=-\gamma+\frac{1}{2} \rho_{d}\left(\gamma^{2}+p^{2}\right)+\cdots,
$$

where $a_{0}$ and $r_{0}$ are the scattering length and effective range for the ${ }^{1} S_{0}$ channel and $\gamma$ is the deuteron momentum $\gamma=\sqrt{m_{N} B}$ ( $B$ is the deuteron binding energy) and $\rho_{d}$ is the effective range for the ${ }^{3} S_{1}$ channel. Now, the above renormalization condition allows us to relate the LECs to the effective-range expansion parameters. For the ${ }^{1} S_{0}$-channel, this procedure leads to $\sigma_{s}=-1$,

$$
y_{s}=\frac{2}{m_{N}} \sqrt{\frac{2 \pi}{r_{0}}}, \quad D_{s}(p)=\frac{m_{N} r_{0}}{2} \frac{1}{\frac{1}{a_{0}}+i p-\frac{1}{2} r_{0} p^{2}} .
$$

For the deuteron channel, one has $\sigma_{t}=-1$ and

$$
y_{t}=\frac{2}{m_{N}} \sqrt{\frac{2 \pi}{\rho_{d}}}, \quad D_{t}(p)=\frac{m_{N} \rho_{d}}{2} \frac{1}{\gamma+i p-\frac{1}{2} \rho_{d}\left(\gamma^{2}+p^{2}\right)}=\frac{Z_{d}}{E+B}+\cdots
$$

where $Z_{d}$ is the wave function normalization factor of the deuteron at the pole $E=-B$, and the ellipsis in Eq. (14) denotes corrections that are finite or vanish at $E=-B$. Thus one has $[15]$

$$
Z_{d}=\frac{\gamma \rho_{d}}{1-\gamma \rho_{d}}
$$

This $Z_{d}$ is equal to the asymptotic $S$-state normalization constant discussed in Introduction. It is to be noted that the order of the LECs $y_{s, t}$ is now of $Q^{1 / 2}$, and the deuteron state is described by the dressed dibaryon propagator that contains two-nucleon loops as well as the bare ${ }^{3} S_{1}$ dibaryon.

The $d d A$ vertex in Eq. (7) contains a LEC $l_{1 A}$, which is associated with the LEC, $\hat{d}^{R}$ or $L_{1 A}$, appearing in the contact-type two-nucleon-axial-vector vertex. It is not obvious how to relate $l_{1 A}$ to $\hat{d}^{R}$ or $L_{1 A}$, because the dimensions of these LECs are different; $l_{1 A}$ is a dimensionless quantity, whereas, for instance, $L_{1 A}$ is measured in units of $\mathrm{fm}^{3}$ because of two more baryon fields involved in the vertex. However, a relation between $l_{1 A}$ and $L_{1 A}$ is discussed in Refs. [28, 30]. We employ here the assumption proposed in Ref. [37] that $l_{1 A}$ involves both LO and subleading-order parts. The LO part is fixed from the one-body $N N A$ interaction vertex, which is proportional to $g_{A}$ and the factor $\left(\frac{r_{0}+\rho_{d}}{2 \sqrt{r_{0} \rho_{d}}}\right) \simeq 1.024$, which has been introduced so as to reproduce the result of effective range theory. The subleading term $l_{1 A}$ represents a two-body interaction and its value is fixed by using the ratio of the two- and one-body amplitudes (see below).

\section{Cross section and numerical results}

We calculate the $n n$-fusion amplitude by adding the contributions from diagrams (a), (b) and (c) in Fig. 3. Since the initial two neutrons are in the ${ }^{1} S_{0}$ state, the dressed 


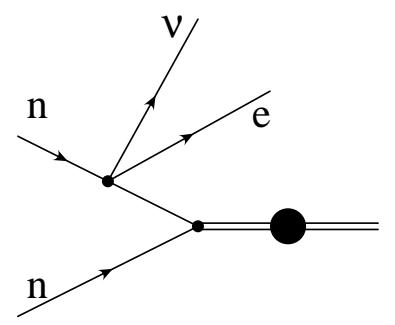

(a)

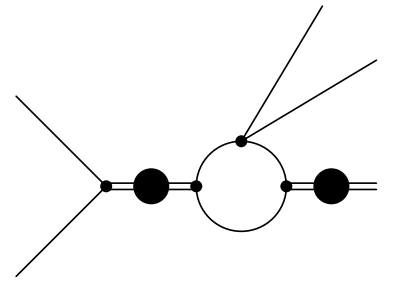

(b)

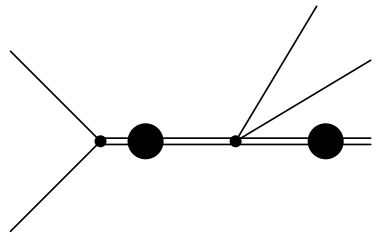

(c)

Figure 3: Diagrams for neutron-neutron fusion, $n n \rightarrow d e \nu$, up to next-to leading order.

dibaryon propagator in diagrams (b) and (c) is limited to be $D_{s}(p)$ (the contribution from $D_{t}(p)$ is highly suppressed due to the orthogonality between the initial scattering and final bound ${ }^{3} S_{1}$ states). Meanwhile, the final deuteron state is described by the wavefunction normalization factor $\sqrt{Z_{d}}$. Thus we have the $n n$-fusion amplitude

$$
\begin{aligned}
A^{(a+b+c)}\left({ }^{1} S_{0}\right)= & \vec{\epsilon}_{(d)}^{*} \cdot \vec{\epsilon}_{(l)} G_{F} V_{u d} \sqrt{\frac{2 \pi \gamma}{1-\gamma \rho_{d}}} \frac{2}{m_{N}} \frac{a_{0}^{n n} g_{A}}{E_{e}^{\max }} \\
& \times\left[\sqrt{m_{N} E_{e}^{\max }}-\frac{1}{a_{0}^{n n}}-\frac{1}{4}\left(r_{0}^{n n}+\rho_{d}\right) m_{N} E_{e}^{\max }-\frac{E_{e}^{\max }}{2 g_{A}} l_{1 A}\right],
\end{aligned}
$$

where $\vec{\epsilon}_{(d)}^{*}$ is the spin polarization vector of the deuteron and $\vec{\epsilon}_{(l)}$ is the spatial part of the lepton current $l^{\mu}=\left(\epsilon_{(l)}^{0}, \vec{\epsilon}_{(l)}\right)$. Note that the amplitude in Eq. (16) is proportional to $a_{0}^{n n}$ and $g_{A}$. It also depends on $l_{1 A}$. We remark that the amplitude obtained above is similar to that for low-energy $n p \rightarrow d \gamma$ capture calculated in the effective-range expansion approach and in an NLO dEFT calculation; the $n p \rightarrow d \gamma$ process involves the same partial waves, the initial ${ }^{1} S_{0}$ wave and the final ${ }^{3} S_{1}$ deuteron state. Thus, by changing $\sqrt{m_{N} E_{e}^{\max }}$ in the bracket in Eq. (16) to $\sqrt{m_{N} B}$, one obtains an expression analogous to the amplitude for $n p \rightarrow d \gamma$ (see, e.g., Eq. (39) in Ref. [37]).

The differential cross section for $n n$-fusion is now easily obtained. We include here the Fermi function, which describes the Coulomb interaction between the out-going electron and the deuteron. Furthermore, we take into account the radiative corrections of $\mathcal{O}(\alpha)$ calculated for the one-body transition diagrams [38]; here $\alpha$ is the fine structure constant. These effects need to be incorporated in order to achieve accuracy better than $1 \%$ in the calculated cross section. We then arrive at ${ }^{8}$

$$
\begin{aligned}
\frac{d \sigma}{d E_{e}}= & 6 p_{e} E_{e}\left(E_{e}^{\max }-E_{e}\right)^{2} F\left(Z, E_{e}\right)\left[1+\frac{\alpha}{2 \pi} \delta_{\alpha}^{(1)}\right] \\
& \times \frac{1}{v} \frac{G_{V}^{2}}{\pi^{2}}\left(\frac{a_{0}^{n n} g_{A}}{\gamma_{n n}}\right)^{2} \frac{\gamma}{1-\gamma \rho_{d}}\left[1-\frac{1}{\gamma_{n n} a_{0}^{n n}}-\frac{1}{4}\left(r_{0}^{n n}+\rho_{d}\right) \gamma_{n n}-\frac{\gamma_{n n} l_{1 A}}{2 m_{N} g_{A}}\right]^{2},
\end{aligned}
$$

\footnotetext{
${ }^{8}$ We have used the relation $\sum_{\text {spin }}\left|\vec{\epsilon}_{(d)}^{*} \cdot \vec{\epsilon}_{(l)}\right|^{2}=6-2 \beta y$, where $\beta=\left|\vec{p}_{e}\right| / E_{e}$ and $y=\hat{p}_{e} \cdot \hat{p}_{\nu}$.
} 
where $F\left(Z, E_{e}\right)$ is the Fermi function defined by $F\left(Z, E_{e}\right)=x /(1-\exp (-x))$ with $x=$ $2 \pi \alpha Z / \beta ; \beta$ is the electron velocity $\beta=\left|\vec{p}_{e}\right| / E_{e}$, and $\gamma_{n n}=\sqrt{m_{N} E_{e}^{\max }}$. Furthermore,

$$
G_{V}^{\prime 2}=\left(G_{F} V_{u d}\right)^{2}\left[1+\frac{\alpha}{2 \pi} e_{V}^{R}\right]
$$

where $e_{V}^{R}$ is an LEC that appears in calculating radiative corrections. Finally $\delta_{\alpha}^{(1)}$ is the radiative correction of $\mathcal{O}(\alpha),{ }^{9}$

$$
\begin{aligned}
\delta_{\alpha}^{(1)}= & 3 \ln \left(\frac{m_{p}}{m_{e}}\right)+\frac{1}{2}+\frac{1+\beta^{2}}{\beta} \ln \left(\frac{1+\beta}{1-\beta}\right)-\frac{1}{\beta} \ln ^{2}\left(\frac{1+\beta}{1-\beta}\right)+\frac{4}{\beta} L\left(\frac{2 \beta}{1+\beta}\right) \\
& +4\left[\frac{1}{2 \beta} \ln \left(\frac{1+\beta}{1-\beta}\right)-1\right]\left[\ln \left(\frac{2\left(E_{e}^{\text {max }}-E_{e}\right)}{m_{e}}\right)+\frac{1}{3}\left(\frac{E_{e}^{\text {max }}-E_{e}}{E_{e}}\right)-\frac{3}{2}\right] \\
& +\left(\frac{E_{e}^{\text {max }}-E}{E_{e}}\right)^{2} \frac{1}{12 \beta} \ln \left(\frac{1+\beta}{1-\beta}\right)
\end{aligned}
$$

with $L(x)=\int_{0}^{x} \frac{d t}{t} \ln (1-t)$.

Eqs. (17) and (18) involve two LECs, $e_{V}^{R}$ and $l_{1 A}$, which need to be fixed. The LEC $e_{V}^{R}$ can be fixed using the experimental value of the neutron lifetime $\tau$ and the axial current coupling $g_{A}$. We use the expression for $\tau$ given in Ref. [38] and employ the experimental values, $\tau=885.7(8)$ sec and $g_{A}=1.2695(29)$ quoted in PDG2004 [40]. The LEC, $l_{1 A}$, can in principle be fixed by applying dEFT to the $A=3$ nuclear systems and using the tritium $\beta$-decay rate to constrain $l_{1 A}$, a procedure similar to the one adopted in the $\mathrm{EFT}^{*}$ calculations [19]. However, a dEFT calculation for the three-nucleon system with an external weak current is yet to be done. We therefore make here partial use of the results obtained in the EFT* calculations [19]. According to Ref. [19], the cross section for charged-current weak-interaction processes in the two-nucleon system receives about 2 percent corrections from the (two-body) exchange current; see, e.g., Eq. (29) in Ref. [19]. ${ }^{10}$ We may then fix $l_{1 A}$ by imposing the condition that the term involving $l_{1 A}$ should enhance the $\bar{\nu}_{e} d \rightarrow e^{+} n n$ cross section by $2 \%$ at the initial neutrino energy $E_{\nu}=20 \mathrm{MeV}$ [41]. This requirement leads to

$$
\frac{\alpha}{2 \pi} e_{V}^{R}=(2.01 \pm 0.40) \times 10^{-2}, \quad l_{1 A}=-0.33 \pm 0.03
$$

\footnotetext{
${ }^{9}$ We note that $\delta_{\alpha}^{(1)}$ is not exactly the same as the outer radiative correction $g\left(E_{0}, E\right)$ given in [39], owing to a slightly different renormalization scheme employed calculating $\delta_{\alpha}^{(1)}$ [38]; these two quantities are related as $\delta_{\alpha}^{(1)}=g\left(E_{0}, E\right)+5 / 4$. This feature leads to a difference of $\frac{5}{4} \frac{\alpha}{2 \pi} \simeq 1.45 \times 10^{-3}$ in each of the subsequent expressions.

${ }^{10}$ Since each scheme of EFT comes with its specific definition of the field variables, the comparison of the strengths of the two-body contributions in different schemes involves subtleties. In the present exploratory study, however, we simply use the result of EFT* to fix the value of $l_{1 A}$ in dEFT. We will give a brief remark on this point later in the text. It is worth mentioning here that the precision of the calculated cross section turns out to be controlled by the errors in the currently available experimental values of $a_{0}^{n n}$ and $r_{0}^{n n}$.
} 
Here we have used $G_{F}=1.16637(1) \times 10^{-5} \mathrm{GeV}^{-2}$ determined from muon decay [40], and $V_{u d}=0.9738(4)$ deduced from the $0^{+} \rightarrow 0^{+}$nuclear $\beta$-decays [42]. The quoted errors in $e_{V}^{R}$ are dominated by the uncertainties in $g_{A}$, while the errors in $l_{1 A}$ reflect the $\sim 0.2 \%$ error in the calculated $\nu$ - $d$ cross sections, which in turn are associated with the errors in the experimental value of the tritium $\beta$-decay rate.

We also need to specify the $n n$ scattering length and effective range. Their current experimental values are (see, e.g., Ref. [43])

$$
a_{0}^{n n}=-18.5 \pm 0.4 \quad[\mathrm{fm}], \quad r_{0}^{n n}=2.80 \pm 0.11 \quad[\mathrm{fm}] .
$$

Thus there are $\sim 2 \%$ and $\sim 4 \%$ uncertainties in $a_{0}^{n n}$ and $r_{0}^{n n}$, respectively. We will discuss later the consequences of these uncertainties. We do not include in Eq. (17) the $1 / m_{N}$ corrections or nuclear-dependent (two-body part) $\mathcal{O}(\alpha)$ corrections, the contribution of which are about $0.1 \%$.

Having specified the LECs and other parameters appearing in our formalism, we are now in a position to calculate the cross section and electron energy spectrum for $n n$ fusion. We find it convenient to present our numerical results at a certain specified incident neutron energy; the cross sections at other energies can be readily obtained by using the $1 / v$ law. So we consider ultra-cold neutrons (UCN). A typical temperature for UCN is $T_{U C N} \sim 1 \mathrm{mK}$, and the corresponding average velocity is $v_{U C N} \sim 5 \mathrm{~m} / \mathrm{sec}$. So, for the sake of definiteness, we may consider a head-on collision of two neutrons each moving with $v_{U C N}=5 \mathrm{~m} / \mathrm{sec}$; thus in the CM system of these two neutrons $v=2 v_{U C N} \sim 10$ $\mathrm{m} / \mathrm{sec}$. The numerical results given below correspond to this kinematics.

In Fig. 4, we plot the calculated electron energy spectrum, $d \sigma / d E_{e}$, as a function of $E_{e}$. As mentioned, the electrons with $E_{e}>\delta_{N}=1.29 \mathrm{MeV}$ can in principle be distinguishable from the electrons from neutron $\beta$-decay. Since the amplitude in Eq. (16) is independent of $E_{e}$, the shape of the electron energy spectrum in Fig. 4 is determined mainly by the phase factor and the $\mathcal{O}(\alpha)$ corrections coming from the Fermi function and the radiative correction, $\delta_{\alpha}^{(1)}$, in Eq. (19).

We also calculate the total cross section $\sigma$ as well as $\sigma_{c u t}$, the latter being the differential cross section integrated over $E_{e}>\delta_{N} ;$ viz.

$$
\sigma=\int_{m_{e}}^{E_{e}^{\max }} d E_{e} \frac{d \sigma}{d E_{e}}, \quad \sigma_{c u t}=\int_{\delta_{N}}^{E_{e}^{\max }} d E_{e} \frac{d \sigma}{d E_{e}}
$$

The results are

$$
\sigma=(38.6 \pm 1.5) \times 10^{-40}\left[\mathrm{~cm}^{2}\right], \quad \sigma_{\text {cut }}=(30.2 \pm 1.2) \times 10^{-40}\left[\mathrm{~cm}^{2}\right] .
$$

Since there is no $E_{e}$-dependence in the transition amplitude in Eq. (16), the relative errors in $\sigma$ and $\sigma_{\text {cut }}$ are the same. The $\sim 4 \%$ uncertainties in the cross sections in Eq. (23) mainly come from the errors in the experimental values of $a_{0}^{n n}$ and $r_{0}^{n n}$, Eq. (21). We note that the uncertainties in $a_{0}^{n n}$ and $r_{0}^{n n}$ affect the cross sections to about the same extent; the $\sim 2.2 \%$ error in $a_{0}^{n n}$ leads to $\sim 3.4 \%$ uncertainty, and the $\sim 4 \%$ error in $r_{0}^{n n}$ to $\sim 1.9 \%$ uncertainty. 


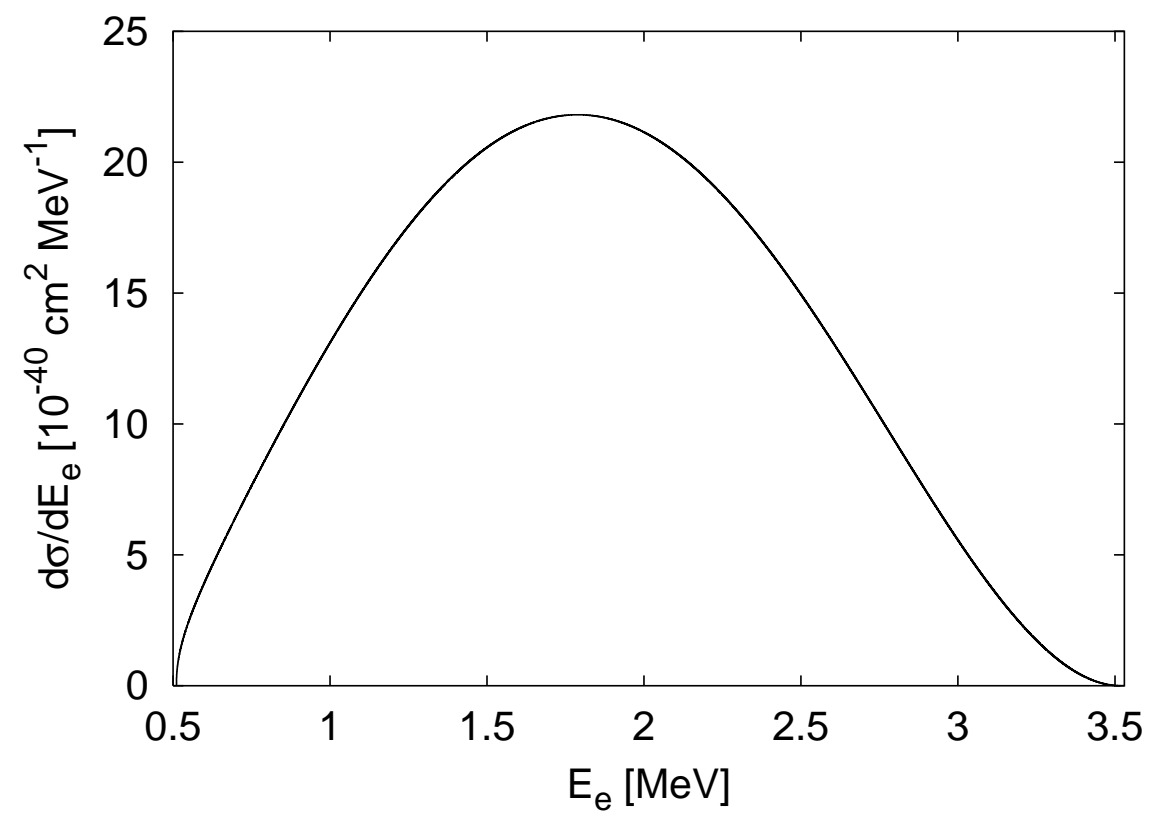

Figure 4: Spectrum of the electrons from neutron-neutron fusion, $n n \rightarrow d e \nu$.

\section{Discussion and conclusions}

In this paper we studied the $n n$-fusion process at low energies employing the pionless EFT that incorporates the dibaryon fields (pionless dEFT). The electron energy spectrum and the integrated cross sections were calculated up to NLO. We included the $\mathcal{O}(\alpha)$ radiative corrections calculated for the one-body transition contributions. Our formalism involves the two LECs, $e_{V}^{R}$ and $l_{1 A}$. The former is associated with the inner radiative correction in $\beta$-decay, and the latter with the short-range two-nucleon electroweak interaction. We fixed these LECs with sufficient accuracy for our present purposes in the following manner. The LEC, $e_{V}^{R}$, is fixed using the experimental data on neutron $\beta$-decay. The LEC, $l_{1 A}$, is constrained with the use of the results obtained in the EFT* calculations in the literature, in which this short-range electroweak effect was determined from the tritium $\beta$-decay rate. Once this is done, we can calculate the $n n$-fusion rate with about $4 \%$ accuracy. The uncertainties in our theoretical estimates are dominated by the existing uncertainties in the measured values of the $n n$ scattering length $a_{0}^{n n}$ and effective range $r_{0}^{n n}$.

In view of the enormous difficulty of observing $n n$-fusion, elaborate calculations of its cross section are not warranted at the present stage. We have however presented a detailed treatment of $n n$-fusion, because the same formalism can be used for the other related processes for which high-precision calculations are certainly needed. The remainder of this section is written in the same spirit.

To fix the value of $e_{V}^{R}$, we have used here the current standard values of $G_{F}, V_{u d}, \tau$, and 
$g_{A}$. We note, however, that one of the main purposes of high-precision measurements of neutron $\beta$-decay is to deduce the accurate value of $V_{u d}$ avoiding nuclear-model dependence. It is therefore important to determine $e_{V}^{R}$ through other experiments. ${ }^{11}$ Moreover, a recent measurement of the neutron lifetime $\tau$ [46] reported a value that differs from the existing world average value by 6.5 standard deviations. A new precise measurement of neutron $\beta$-decay currently under planning is essential to clarify this discrepancy and to determine the value of $V_{u d}$. (A change in the $n n$-fusion cross section due to the new experimental value of $\tau$ is smaller than the uncertainties due to the limited accuracy of $a_{0}^{n n}$ and $r_{0}^{n n}$.)

We have fixed the value of $l_{1 A}$ using the result of the EFT* and potential model calculations for the tritium $\beta$-decay. It is an important future task to determine $l_{1 A}$ within the framework of dEFT itself. For cases that do not involve external currents, there exists work in which dEFT is applied to the three-nucleon systems [47]. We need to extend this type of work to cases that include external currents. Meanwhile, with the use of the value of $l_{1 A}$ deduced in the present work, we can carry out dEFT calculations of the $p p$-fusion process and $\nu d$ reactions with no free parameters. As mentioned earlier, a relation between $l_{1 A}$ and $L_{1 A}$ was discussed in Refs. [28, 30]. The relation given in Ref. [28] leads to $L_{1 A}=-0.26 \pm 0.11 \mathrm{fm}^{3}$, while that derived in Ref. [30] gives $L_{1 A}=1.18 \pm 0.11$ $\mathrm{fm}^{3}$. The indicated errors are consistent with those in the value of $L_{1 A}$ deduced from the tritium $\beta$-decay, $L_{1 A}=4.2 \pm 0.1 \mathrm{fm}^{3}$ [27], but the central values are smaller. It is possible that this discrepancy stems from the different expansion schemes used in these calculations.

We remark that the contribution from the $l_{1 A}$ term probably can play a significant role in other processes, such as $\pi d \rightarrow n n \gamma$ and $\mu d \rightarrow n n \nu$, which are used for deducing $a_{0}^{n n}$ and $r_{0}^{n n}$. Recent (pionful) EFT calculations for $\pi d \rightarrow n n \gamma[6]$ and $\gamma d \rightarrow n n \pi$ [48] indicate that the Kroll-Ruderman (KR) term is important for these reactions. Since the KR term is related to the axial-vector couping constant $g_{A}$ through chiral symmetry, and since $g_{A}$ is known to be modified by the multi-nucleon effects, it is likely to be important to include the short-range two-body effects in analyzing the $\pi d \rightarrow n n \gamma$ and $\gamma d \rightarrow n n \pi$ processes.

\section{Acknowledgments}

SA thanks Y. Li, A. Gardestig, and S. X. Nakamura for communications. This work is supported in part by the Natural Science and Engineering Research Council of Canada and by the United States National Science Foundation, Grant No. 0140214.

\section{References}

[1] I. Šlaus, Y. Akaishi, and H. Tanaka, Phys. Rep. 173 (1989) 257.

\footnotetext{
${ }^{11}$ As discussed in Ref. [44], a term (known as the $C$ term) in the inner radiative correction [45] has model dependence because, when the loop diagrams involve the axial current, one cannot exactly match the calculations based on the quark degrees of freedom (in short range) with those based on the hadron degrees of freedom (in long range). It seems worthwhile to calculate the $C$ term in other models and estimate the model dependence of the existing treatments of the inner radiative corrections.
} 
[2] G. A. Miller, B. M. K. Nefkens and I. Šlaus, Phys. Rep. 194 (1990) 1.

[3] C. R. Howell et al., Phys. Lett. B 444 (1998) 252.

[4] D. E. González Trotter et al., Phys. Rev. Lett. 83 (1999) 3788.

[5] V. Huhn et al., Phys. Rev. Lett. 85 (2000) 1190.

[6] A. Gardestig and D. R. Phillips, nucl-th/0501049.

[7] B. E. Crawford et al., J. Phys. G: Nucl. Phys. 30 (2004) 1269.

[8] S. Weinberg, hep-th/9702027.

[9] S. R. Beane et al., in (ed.) M. Shifman, At the Frontier of Particle Physics, Vol.1, 133, World Scientific, Singapore (2001); nucl-th/0008064.

[10] P. F. Bedaque and U. van Kolck, Ann. Rev. Nucl. Part. Sci. 52 (2002) 339.

[11] K. Kubodera, in the proceedings of the KIAS-APCTP International Symposium on Astro-Hadron Physics, Seoul, Korea (November 10-14, 2003), eds. D.-K. Hong et al., World Scientific (Singapore, 2004), p.433 (nucl-th/0404027); K. Kubodera and T.-S. Park, Annu. Rev. Nucl. Part. Sci. 54 (2004) 19 (nucl-th/0402008).

[12] S. Weinberg, Phys. Lett. B 251 (1990) 288.

[13] D. B. Kaplan, M. J. Savage, and M. B. Wise, Phys. Lett. B 424 (1998) 390.

[14] See, e.g., J.-W. Chen, G. Rupak, and M. J. Savage, Nucl. Phys. A 653 (1999) 386 and references therein.

[15] S. R. Beane and M. J. Savage, Nucl. Phys. A 694 (2001) 511.

[16] T.-S. Park, K. Kubodera, D.-P. Min, and M. Rho, Astrophys. J. 507 (1998) 443.

[17] X. Kong and F. Ravndal, Nucl. Phys. A 656 (1999) 421; X. Kong and F. Ravndal, Phys. Lett. B 470 (1999) 1; X. Kong and F. Ravndal, Phys. Rev. C 64 (2001) 044002.

[18] M. Butler and J.-W. Chen, Phys. Lett. B 520 (2001) 87.

[19] T.-S. Park et al., Phys. Rev. C 67 (2003) 055206 (nucl-th/0208055); see also T.-S. Park et al., nucl-th/0106025 and nucl-th/0107012.

[20] S. Nakamura, T. Sato, V. Gudkov, and K. Kubodera, Phys. Rev. C 63 (2001) 034617.

[21] S. Nakamura et al., Nucl. Phys. A 707 (2002) 561.

[22] M. Butler and J.-W. Chen, Nucl. Phys. A 675 (2000) 575.

[23] M. Butler, J.-W. Chen, and X. Kong, Phys. Rev. C 63 (2001) 035501. 
[24] S. Ando, Y.-H. Song, T.-S. Park, H. W. Fearing, and K. Kubodera, Phys. Lett. B 555 (2003) 49.

[25] S. X. Nakamura, Prog. Theor. Phys. 114 (2005) 713.

[26] M. Butler, J.-W. Chen and P. Vogel, Phys. Lett. B 549 (2002) 26.

[27] J.-W. Chen, K. M. Heeger, and R. G. Hamish Robertson, Phys. Rev. C 67 (2003) 025801.

[28] W. Detmold and M. J. Savage, Nucl. Phys. A 743 (2004) 170.

[29] S. Ando, T.-S. Park, F. Myhrer, and K. Kubodera, Phys. Lett. B 533 (2002) 25.

[30] J.-W. Chen, T. Inoue, X. Ji, and Y. Li, nucl-th/0506001.

[31] P. Kammel, nucl-th/0304019.

[32] M. Rho, AIP Conf. Proc. 494 (1999) 391; nucl-th/9908015.

[33] D. R. Phillips and T. D. Cohen, Nucl. Phys. A 668 (2000) 45.

[34] D. R. Phillips, G. Rupak, and M. J. Savage, Phys. Lett. B 473 (2000) 209.

[35] D. B. Kaplan, Nucl. Phys. B 494 (1997) 471.

[36] B. F. Bedaque and U. van Kolck, Phys. Lett. B 428 (1998) 221.

[37] S. Ando and C. H. Hyun, Phys. Rev. C 72 (2005) 014008, nucl-th/0407103.

[38] S. Ando, H. W. Fearing, V. Gudkov, K. Kubodera, F. Myhrer, S. Nakamura, and T. Sato, Phys. Lett. B 595 (2004) 250.

[39] A. Sirlin, Phys. Rev. 164 (1967) 1767.

[40] S. Eidelman et al., Phys. Lett. B 592 (2004) 1.

[41] S. Ando and K. Kubodera, in progress.

[42] J. C. Hardy and I. S. Towner, Phys. Rev. C 71 (2005) 055501.

[43] G. F. de Téramond and B. Gabioud, Phys. Rev. C 36 (1987) 691.

[44] M. Fukugita and T. Kubota, Acta Phys. Polon. B 35 (2004) 1687.

[45] I. S. Towner, Nucl. Phys. A 540 (1992) 478.

[46] A. Serebrow et al., Phys. Lett B 605 (2005) 72.

[47] P. F. Bedaque, H.-W. Hammer, and U. van Kolck, Nucl. Phys. A 676 (2000) 357.

[48] V. Lensky et al., nucl-th/0505039. 